This is an Accepted Manuscript of

G. Mazzanti, "Including the calculation of transient electric field in the life estimation of HVDC cables subjected to load cycles"

in:

IEEE Electrical Insulation Magazine, vol. 34, no. 3, pp. 27-37, May-June 2018

The final published version is available online at:

https://doi.org/10.1109/MEl.2018.8345358

Rights / License:

The terms and conditions for the reuse of this version of the manuscript are specified in the publishing policy. For all terms of use and more information see the publisher's website.

This item was downloaded from IRIS Università di Bologna (https://cris.unibo.it/)

When citing, please refer to the published version. 


\section{Including the Calculation of Transient Electric Field in the Life Estimation of HVDC Cables Subjected to Load Cycles}

Key words: DC power transmission, HVDC insulation, life estimation, transient DC electric field, testing

Giovanni Mazzanti

Department of Electrical, Electronic and Information Engineering "Guglielmo Marconi”,

Alma Mater Studiorum - University of Bologna

Viale Risorgimento 2

Bologna, I-40136, Italy

The calculation of transient DC electric field is included in a proposed procedure for life estimation of HVDC cables under time-varying electro-thermal stress associated with load cycling.

\section{Introduction}

The need for long subsea power links, the advantages of HVDC (High Voltage Direct Current) transmission and the concerns about overhead lines are promoting a booming growth of submarine and land DC cable lines worldwide [1]. In particular the performance of HVDC extruded cable systems has been improved significantly in recent years. The ratings have increased, with bipolar DC extruded cable systems already in service at voltage and power levels up to $320 \mathrm{kV}$ and $1 \mathrm{GW}$ respectively [2]-[5], and DC extruded cable systems being qualified at voltage ratings up to $525 \mathrm{kV}$ [6],[7].

At such ratings the long term performance of DC cable systems is critical, but uncertain, not only for the accessories (joints and terminations) [8], but for the cable itself. The prequalification (PQ) test, prescribed in CIGRÉ Technical Brochure 496 [9] for simulating service life so as to evaluate "the long-term performances of the cable system", is a crucial step in the qualification of DC cable systems. In addition, models for estimating the life, i.e., time-to-failure, and reliability of full-size HVDC extruded cables in service are very useful tools at the design stage, for selecting appropriate operating stress levels, and at the testing stage, for completing and interpreting the information from the PQ test.

Estimating the service life is much more difficult for DC than for AC cables [1],[10]. In AC cables the electric field $E_{A C}$ depends on the dielectric permittivity $\varepsilon$, and can be taken as constant irrespective of load cycle. On the contrary, in DC cables the electric field $E_{D C}$ depends on the volume electrical conductivity $\sigma$ of the dielectric, which in turn depends on the temperature $T$ and $E_{D C}$ [11],[12]. Load cycling therefore implies a time dependence of the electro-thermal stress, which makes the development of a life model for DC cables under load cycling much more difficult than for AC cables.

A procedure for life estimation of HVDC cables under the time-varying electro-thermal stress associated with load cycles was proposed for the first time in [13]. This procedure relies on a few approximations. The most severe is that the DC field $E_{D C}(r, t)$, at radius $r$ within the cable insulation and at time $t$ during the thermal transients associated with load cycles, is calculated using Eoll's formula for the DC electric field $E_{D C}(r)$ in steady-state [14]. This approximation was called the "quasi-steady" DC field hypothesis in [13], since it is equivalent to assuming that the field varies slowly between one steady (or equilibrium) profile to another. The aim of the present paper is to improve the procedure for life estimation of DC cables subjected to load cycles, by replacing the quasi-steady hypothesis with an approach which involves solving Maxwell's equations in accordance with the guidelines given in [15].

It must be emphasized that, compared to the algorithm given in [15], the proposed procedure assumes that negligible heat is generated in the insulation, which then enables decoupling the calculation of temperature from that of field. Clearly this holds for well-designed cables, but it is not true for thermal break- 
down, analysis of which lies outside the scope of the proposed procedure. However, the proposed procedure provides an a-posteriori check of the heat generated in the dielectric. If such heat is considered nonnegligible, the temperature is updated and the field is recalculated.

\section{Procedure for DC Cable Life Estimation under Load Cycles}

The procedure for life estimation of HVDC cables subjected to load cycles proposed in [13] resembles that given in [16], [17] for HVAC cables, but with some major differences which account for the characteristics of the DC electric field. The procedure given in [13] consists of the following blocks:

- Block 1: load cycles are assumed to last for a time $t_{d}=24 \mathrm{~h}$, with known time-dependence $I=f(t)$ of the load current $I$, and cable features such as geometry, materials and voltage are taken as known.

- Block 2: thermal transients associated with load cycles are calculated following the CIGRÉ thermal network guide adapted for DC cables [18]. Thus temperature profiles $T(r, t)$ are calculated covering various radial coordinates within the cable insulation between $r_{i}$ and $r_{o}$, the inner and the outer insulation radii respectively, and at various times $t$ during the cycle.

- Block 3: DC field profiles $E_{D C}(r, t)$ within the cable insulation are calculated using Eoll's formula, where the temperature drop across the insulation thickness at time $t, \Delta T(t)=T\left(r_{i, t}\right)-T\left(r_{o}, t\right)$, is computed via the temperature profiles $T(r, t)$ determined in Block 2.

- Block 4: the life fractions $L F_{D C}(r)$ lost during each cycle $t_{d}$ at each radius $r$ within the insulation are calculated using Miner's law of cumulated aging [19], and DC cable life $L_{D C}$ is estimated as the insulation life at the most severely-stressed point. More precisely

$$
\begin{gathered}
L F_{D C}(r)=\int_{0}^{t_{d}} d t / L\left[E_{D C}(r, t), T(r, t)\right]=1 / K_{D C}(r) \\
L_{D C}(r)=t_{d} \times K_{D C}(r) \\
L_{D C}=\min \left\{L_{D C}(r), r \in\left[r_{i}, r_{o}\right]\right\}=\min \left\{t_{d} \times K_{D C}(r), r \in\left[r_{i}, r_{o}\right]\right\}
\end{gathered}
$$

where:

- $\quad L\left[E_{D C}(r, t), T(r, t)\right]=$ cable insulation life at field $E_{D C}(r, t)$ and temperature $T(r, t)$

- $\quad K_{D C}(r)=$ number of cycles-to-failure at each radius $r$ within cable insulation

- $\quad L_{D C}(r)=$ insulation life under load cycles at each radius $r$ within cable insulation.

(3) shows that cable life $L_{D C}$ under load cycling is the minimum value of insulation life $L_{D C}(r)$, where $\mathrm{r}$ is the insulation radius at which maximum stress occurs.

This procedure works satisfactorily for any expression used to describe the dependence of $\sigma$ on $E_{D C}$ and $T$, provided it is valid for DC cable insulation. The experimentally-verified expression used in the procedure is [1],[2],[15]:

$$
\sigma\left(T, E_{D C}\right)=\sigma_{0} \exp \left[a\left(T-T_{0}\right)+b\left(E_{D C}-E_{0}\right)\right]
$$

where $a$ and $b$ are respectively the temperature and electric stress coefficients of the volume electric conductivity $\sigma$ of the dielectric, and $\sigma_{0}$ is the conductivity at $T=T_{0}$ and $E_{D C}=E_{0} . T_{0}$ and $E_{0}$ are reference values of field and temperature respectively.

The procedure also works satisfactorily for any electrothermal life model used to estimate $L\left[E_{D C}(r, t), T(r, t)\right]$, provided that it is valid for DC cable insulation, e.g., the physical model given in [20]. The so-called IPM-Arrhenius model [21] is sometimes adopted to estimate $L\left[E_{D C}(r, t), T(r, t)\right]$. It is a combination of the IPM electrical and the Arrhenius thermal life model, and yields

$$
L\left[E_{D C}(r, t), T(r, t)\right]=L_{D}\left[E_{D C}(r, t) / E_{D}(r)\right]^{-\left\{n_{D}-b_{E T}\left[1 / T_{D}(r)-1 / T(r, t)\right]\right\}}\left[E_{D}(r) / E_{0}\right]^{b_{E T}\left[1 / T_{D}(r)-1 / T(r, t)\right]} e^{-B\left[1 / T_{D}(r)-1 / T(r, t)\right]}
$$


where $L_{D}$ is the design value of cable life, $E_{D}(r)$ and $T_{D}(r)$ are respectively the design values of electric field and temperature at a generic coordinate $r, n_{D}=n_{0}-b_{E T}\left(1 / T_{0}-1 / T_{D}\right)$ where $n_{0}$ is the life exponent at temperature $T_{0}, B=\Delta W / k_{B}$, where $\Delta W$ is the activation energy of the main degradation reaction of the insulation material and $k_{B}$ is Boltzmann's constant, and $b_{E T}$ is a positive quantity (whose units are $\mathrm{K}$ ) describing the synergism between electrical and thermal stress (for more details on this synergism see [32]).

The IPM-Arrhenius electrothermal life model was chosen in [13] because the IPM and Arrhenius models are supported by the International Standards applicable to HVDC cable insulation [9], [22], and the IPM-Arrhenius electrothermal life model it is in good agreement with times-to-failure data measured on several insulation materials subjected to various voltage/temperature combinations [21],[23].

\section{Improving the Procedure by incorporating the Calculated Transient DC field}

As pointed out above, Eoll's formula holds only under steady state DC conditions, and even then is only an approximation [1],[15]. Immediately after voltage is applied to a previously-unloaded DC cable, the field distribution in the cable insulation is purely capacitive. The distribution then moves gradually towards a purely resistive distribution, which theoretically it attains only after an infinite time. The intermediate field distributions can be computed numerically using the time-dependent Maxwell's equations [1], [15]

Irrotational field

Ohm's law

Poisson's equation

Current continuity

$$
\begin{gathered}
\mathbf{E}=-\nabla V \\
\mathbf{J}=\sigma \mathbf{E} \\
-\nabla \cdot(\varepsilon \nabla V)=\rho \\
\nabla \cdot \mathbf{J}=-\partial \rho / \partial t
\end{gathered}
$$

where $\mathbf{E}$ and $\mathbf{J}$ are the electric field and current density vectors, $V$ is the electric potential, and $\rho$ is the charge density. (6)-(9) imply that the evolution towards steady-state is governed by a time constant $\tau=\varepsilon / \sigma$ which varies strongly with temperature and electric field, mainly because of the corresponding variation in $\sigma[1],[9]$.

In the proposed procedure, $E_{D C}(r, t)$ during the thermal transients associated with load cycles is obtained by solving (6)-(9) numerically with appropriate initial and boundary conditions, rather than by using Eoll's formula. These initial and boundary conditions are as follows:

(1) load cycles start at $t=0$, each cycle lasting $t_{d}=24 \mathrm{~h}$, with known time-dependence $I=f(t)$ of the load current $I$. Thus, the distribution of cable insulation temperature vs. radius and time, $T(r, t)$, can be determined straightforwardly as illustrated in Block 2 and Block 3 of Section 2;

(2) at $t=0$ a known and constant DC voltage $U_{D C}$ is applied across the cable insulation.

Thus the electric field distribution at $t=0$ is known (purely capacitive field), the conductivity, current density and space charge distributions at $t=0$ are known via (4), (7), (8), respectively. Charge injection from the semicon electrodes of HVDC cables is assumed to follow the Schottky law, since the applied fields are usually less than $100 \mathrm{MV} / \mathrm{m}$ [24]. Consistent with the findings reported in [25] for LDPE flat samples with attached carbon-loaded XLPE semicons, injection of holes at the anode is neglected and injection of electrons at the cathode is treated using the Schottky equation [26]. These assumptions yield the direction of main charge carrier velocity, which is used later for implementing upwind differences in equation (9).

The proposed procedure is thus that described in [13] with the addition of an ad hoc algorithm for the numerical solution of (6)-(9) under the initial and boundary conditions stated above. This algorithm provides the transient electric field $E_{D C}(r, t)$ throughout the insulation during the load cycle duration.

The numerical solution of (6)-(9) is summarized in the flow chart of Figure 1. In the algorithm, space and time derivatives are discretized using the finite difference method. In the current continuity equation (9), forward differences were used for $1^{\text {st }}$ order derivatives in time, and upwind differences (an adaptive finite difference scheme which simulates numerically the direction of main charge carrier velocity) 
- Known whole temperature distribution $T(r, t), r \in\left[r_{i}, r_{o}\right], t \in\left[0, t_{d}\right]$ - Known initial distributions at $t(i=0)=0^{-}$and iteration $k=0$ of:

- Electric field: $E_{D C}(r, t(i), k)=E_{D C}\left(r, 0^{-}\right)$

- Space charge: $\rho(r, t(i), k)=\rho\left(r, 0^{-}\right)$

- Current density: $J(r, t(i), k)=J\left(r, 0^{-}\right)$

- Conductivity: $\sigma(r, t(i), k)=\sigma\left(r, 0^{-}\right)$

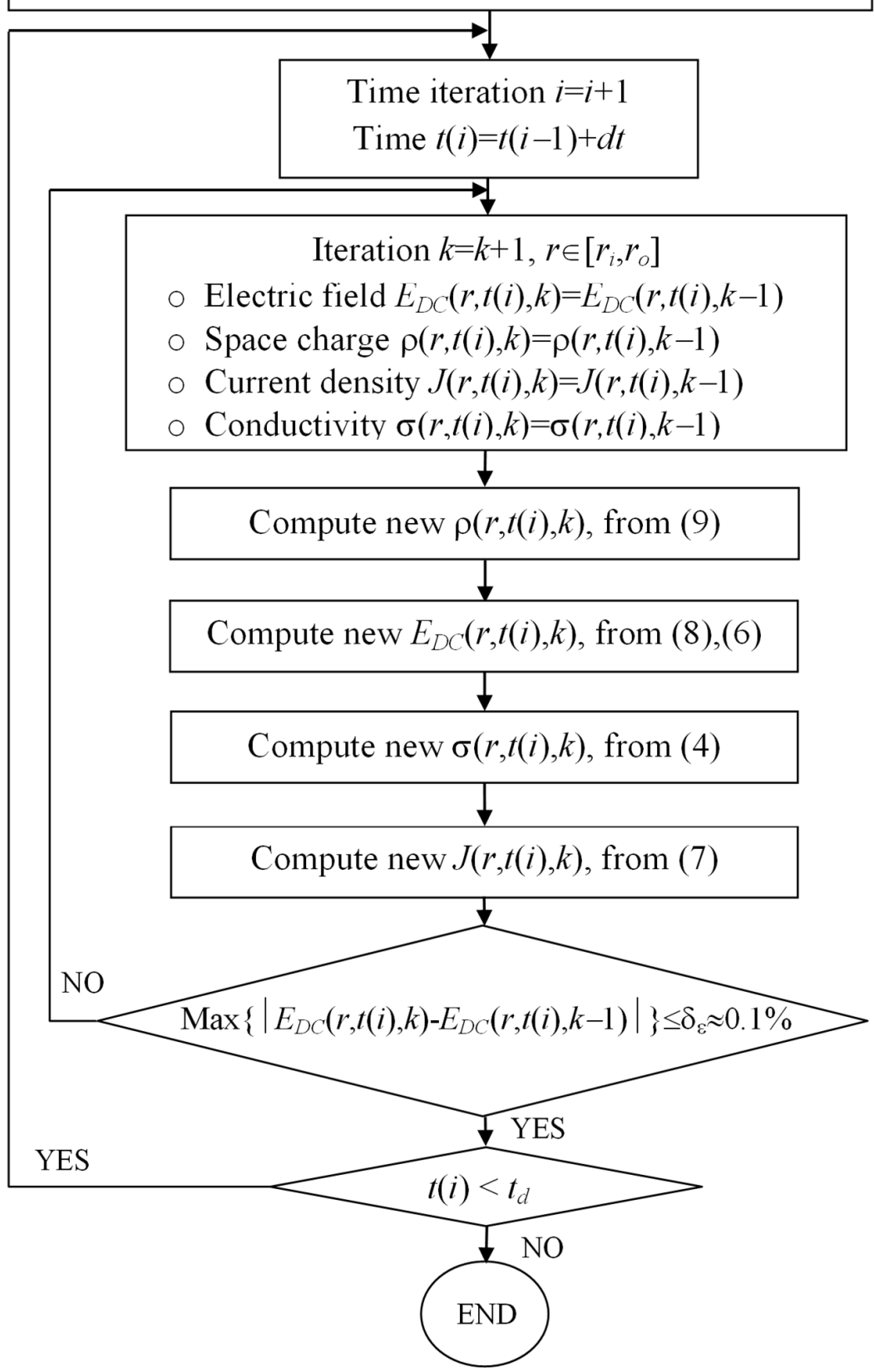

Figure 1. Flow chart of the calculation of transient electric field $E_{D C}(r, t)$ using numerical solution of (6)-(9).
[27],[33]) were used for $1^{\text {st }}$ order derivatives in space. As recommended in [28], the stability of this combination was checked by repeating the calculation for different time steps ( $1 \mathrm{~s}$ to $1 \mathrm{~min}$ ), and for different space steps ( 5 to 50 points over the insulation thickness). Centered finite differences were used in order to solve (7). The flow chart in Figure 1 was validated for the so-called "standard" cable treated in [15], with $U_{0}$ (rated voltage) $=$ $450 \mathrm{kV}, 1600 \mathrm{~mm}^{2}$ copper conductor cross-section, and mass-impregnated non-draining (MIND) insulation [15].

In Figure 2, the lines show the field distribution computed using the algorithm proposed here for the standard cable at different times after the voltage was switched on $(t=0)$, with the lead sheath at a stable temperature $\theta_{S}=35^{\circ} \mathrm{C}$ and a temperature drop $\Delta \theta=15^{\circ} \mathrm{C}$ across the insulation. The circles show the same field distribution computed in [15] at 0 , $25,50,75$ and $100 \%$ of the insulation thickness. The field distribution is shown at $10 \mathrm{~min}$ intervals, with the purely capacitive distribution at $t=0$ and the purely resistive distribution as $\mathrm{t} \rightarrow \infty$. The agreement between the two calculations is very good, the maximum difference at any time and any position being around 1 $\%$. As expected from the large temperature drop across insulation thickness, Figure 2 shows the well-known "field inversion phenomenon" [1],[15], namely a displacement of maximum electric field from the inner to the outer insulation surface as $t \rightarrow \infty$, and the transient field changes from a purely capacitive to a purely resistive distribution. Conversely little field variation is observed at intermediate points [15].

As stated in the Introduction, the field calculation algorithm in the proposed improved procedure is decoupled from the computation of insulation temperature by assuming initially that heat generation in the insulation is negligible, and checking the validity of this assumption later. Figure 2 below and a detailed calculation example reported in [15] show that neglecting ohmic insulation losses is a reasonable hypothesis for a standard MIND cable under typical ser- 
vice and testing conditions. The same assumption holds for the extruded cable discussed below.

\section{Comparison of the Procedures}

We now present some results obtained by applying the original and the improved procedure to one of the two cables considered in [13], i.e., a land XLPE-insulated HVDC cable for use with Voltage-Source Converters (VSC), with typical maximum voltage and power ratings of $320 \mathrm{kV}$ and $1000 \mathrm{MW}$ respectively [4], [5]. The main design parameters are conductor cross section $S=1600 \mathrm{~mm}^{2}$ (copper), DC-XLPE insulation with thickness $s \approx 18 \mathrm{~mm}$, rated current $I_{D}=1727 \mathrm{~A}$, rated conductor temperature $T_{D}=70{ }^{\circ} \mathrm{C}$. The values of the insulation life model parameters in (1)-(3),(5) were the same as those adopted in [13]. The design life and life exponent were set to the values prescribed in [9], i.e., $L_{D}=40 \mathrm{y}$ and $n_{D}=10$. The parameter $B$ was set at $12430 \mathrm{~K}$, a typical value for XLPE [16],[17]. The synergism parameter between electrical and thermal stress $b_{E T}$ was set to zero, which yields the maximum effectiveness of electro-thermal stress synergism in reducing insulation life [21]. The parameters appearing in the CIGRÉ transient thermal network calculation [18] were chosen in agreement with Standard IEC 60287 [29], e.g., the thermal resistivity of the insulation $\rho_{T, d}$ was assumed to be temperature-independent with a value of $3.5 \mathrm{~K} \mathrm{~m} \mathrm{~W}^{-1}$.

As the DC field profiles are very sensitive to the volume electrical conductivity $\sigma$ of the insulation [11],[12], the comparison of the improved procedure with the original procedure includes a parametric sensitivity analysis of the transient DC field profiles to $\sigma$. The sensitivity analysis illustrates the magnitude of changes in the field profiles, and in the life estimates, when $\sigma$ varies within its typical range for the XLPEinsulation of DC cables. The analysis involves calculating the transient field with three different pairs of values of the exponents $a$ and $b$ in (4). These are $a_{L}, b_{L}$ (low set), $a_{M}, b_{M}$ (medium set) and $a_{H}, b_{H}$ (high set), listed in Table 1. Since the values for $a$ and $b$ are not easily found in the literature, these three sets were carefully selected from those reported in [12] for crosslinked compounds. The selection criterion was the level of agreement between the values of time constant $\tau=\varepsilon / \sigma$ of the insulation obtained using the three sets of $a$ and $b$, and the typical ranges of $\sigma$ and $\tau$ versus temperature reported in the Appendix of [9] for various materials used for DC extruded cables. A similar analysis based on low, medium and high sets of $a, b$ was reported in [13], but while $a_{M}, b_{M}$ in Table 1 are the same as in [13], $a_{L}, b_{L}$ and $a_{H}, b_{H}$ are significantly lower than those in [13]. The reasons are (a) the high set given in [13] yields much lower values of $\tau$ than those reported in [9], while the high set selected here yields values of $\tau$ at the lower limit of the ranges reported in [9], and (b) the low set given in [13] yields values of $\tau$ within the ranges given in [9], but the low set chosen here enables broadening the analysis to cases of slow variation of field with time, which could not be treated in [13].

The original and improved versions of the procedure were used to estimate the PQ life of the chosen sample cable, i.e., the cable life under the load cycles of the PQ test for HVDC extruded cable systems prescribed in [9]. The load cycles of the PQ test were chosen since, as pointed out in the Introduction, this test is crucial when evaluating the long-term reliability of a cable system. For VSC cables the PQ test consists of 360 days at constant DC voltage magnitude $U_{P Q}=1.45 U_{0}$ (with changes in voltage polarity omitted here for the sake of brevity) grouped in three periods, namely LC (load cycles), HL (high load) and ZL (zero load). LC consists of 160 24-hour load cycles ; in each cycle the conductor temperature is increased from approximately $T_{a}$ (room temperature) to a temperature $\geq T_{D}$ (typically a few ${ }^{\circ} \mathrm{C}$ above $T_{D}$ ) over the first $6 \mathrm{~h}\left(1^{\text {st }}\right.$ step), maintained at or above $T_{D}$ during the next $2 \mathrm{~h}\left(2^{\text {nd }}\right.$ step), and then reduced to approximately $T_{a}$ over the next $16 \mathrm{~h}$ ( $3^{\text {rd }}$ step). HL consists of 80 days of continuous heating such that the conductor temperature reaches $T_{D}$ within the first $8 \mathrm{~h}$ and is maintained at that temperature for the remainder of the HL period. During ZL (120 days) the cable is not heated, i.e., its temperature is $T_{a}$.

To satisfy the protocols given in [9], in the simulations no current is applied in the ZL period. In the HL period a heating current $I_{H}>I_{D}$ is applied so that the conductor reaches temperature $T_{D}$ in 6 hours, and the rated current $I_{D}$ is applied throughout the rest of the period. In the LC period, 160 24-hr load cycles are applied, each consisting of 3 steps lasting $6 \mathrm{~h}, 2 \mathrm{~h}$ and $16 \mathrm{~h}$ and with rms currents $I_{1}=I_{H}, I_{2}=I_{D}, I_{3}=0$. Thus 




Figure 2. Transient field profiles for a standard cable at various times after switching on the voltage. Lines - computation using the proposed algorithm, circles computation given in [15].
Table 1. Sets of values of conductivity coefficients $a, b$ used in the comparison of the original and improved procedures

\begin{tabular}{|c|c|c|}
\hline & $\begin{array}{c}\text { Temperature } \\
\text { coefficient of } \\
\text { el. conductivity } \\
{\left[\mathrm{K}^{-1}\right]}\end{array}$ & $\begin{array}{c}\text { Electric stress } \\
\text { coefficient of } \\
\text { el. conductivity } \\
{[\mathrm{mm} / \mathrm{kV}]}\end{array}$ \\
\hline low set & $a_{L}=0.042$ & $b_{L}=0.032$ \\
\hline medium set & $a_{M}=0.084$ & $b_{M}=0.0645$ \\
\hline high set & $a_{H}=0.101$ & $b_{H}=0.0775$ \\
\hline
\end{tabular}

the conductor temperature reaches $T_{D}$ in a time $\Delta t_{1}=6 \mathrm{~h}$, remains at $T_{D}$ for a time $\Delta t_{2}=2 \mathrm{~h}$, and then falls to $T_{a}$ in a time $\Delta t_{3}=16 \mathrm{~h}$.

\subsection{Results of the Comparison}

We now apply the original and improved versions of the procedure to the sample cable, from Block 2 to Block 4. (Block 1 has already been completed, i.e., the features of the DC cable and the dependence of load current $I$ vs. time $t$ during the three periods LC, HL and ZL are known).

\section{Block 2: Calculation of Transient Temperature Profiles}

The transient temperature $T(r, t)$ is computed at every radius within the insulation of the cable and at every time within the PQ test, using the CIGRÉ transient thermal network model [18]. Figure 3 shows the transient temperature profiles of the sample cable during the daily load cycles of the LC period at 7 positions. The transient temperature profiles are in agreement with the protocols given in [9].

\section{Block 3: Calculation of Transient DC Field Profiles}

The transient DC field profiles $E_{D C}(r, t)$ were computed using the improved procedure, which yielded "exact" profiles, and using the original procedure, which yielded approximate profiles. The calculated transient fields for the LC are shown in Figures 4 and 5 for the sample cable with $a_{L}, b_{L}$, at the same 5 points within the insulation as in Figure 3. Figure 4 shows the exact profiles during the first 5 cycles. Figure 5(a) shows the exact profiles during the $3^{\text {rd }}$ cycle, and Figure $5(\mathrm{~b})$ shows the approximate profiles (identical for each cycle). The exact and approximate profiles are very sensitive to temperature, i.e., to heating/cooling steps and to location within the insulation, and can be explained qualitatively using the transient temperatures shown in Figure 3.

Figure 4 shows that the exact profiles in the $1^{\text {st }}$ cycle differ from those in the following cycles; however, at each of the 5 points within the insulation they stabilize after the second cycle, as shown in Figure 4. Figure 5(a) focuses on the third cycle, in order to provide a more detailed picture of the stabilized exact field profile. At the inner and outer edges of the insulation the exact profiles never reach steady state, even in the hottest part of the LC cycles, namely from $6 \mathrm{~h}$ to $8 \mathrm{~h}$. The reason is that although the temperatures are highest throughout the insulation in the hottest part of the LC cycles, $a_{L}, b_{L}$ have the lowest values shown in Table 1, implying the lowest conductivity and thus the longest time constant $\tau$ (1.4 to $2.4 \mathrm{~h}$ within the insulation), and the longest time to steady state 10 $\tau$ [9] (ranging from 14 to $24 \mathrm{~h}$ ). Of course steady state is not reached in the cold parts of the LC cycles, since low temperature implies low $\sigma$ and high $\tau$. Conversely, Figure 5(b) shows that the approximate profiles are independent of the cycle, which is to be expected be- 

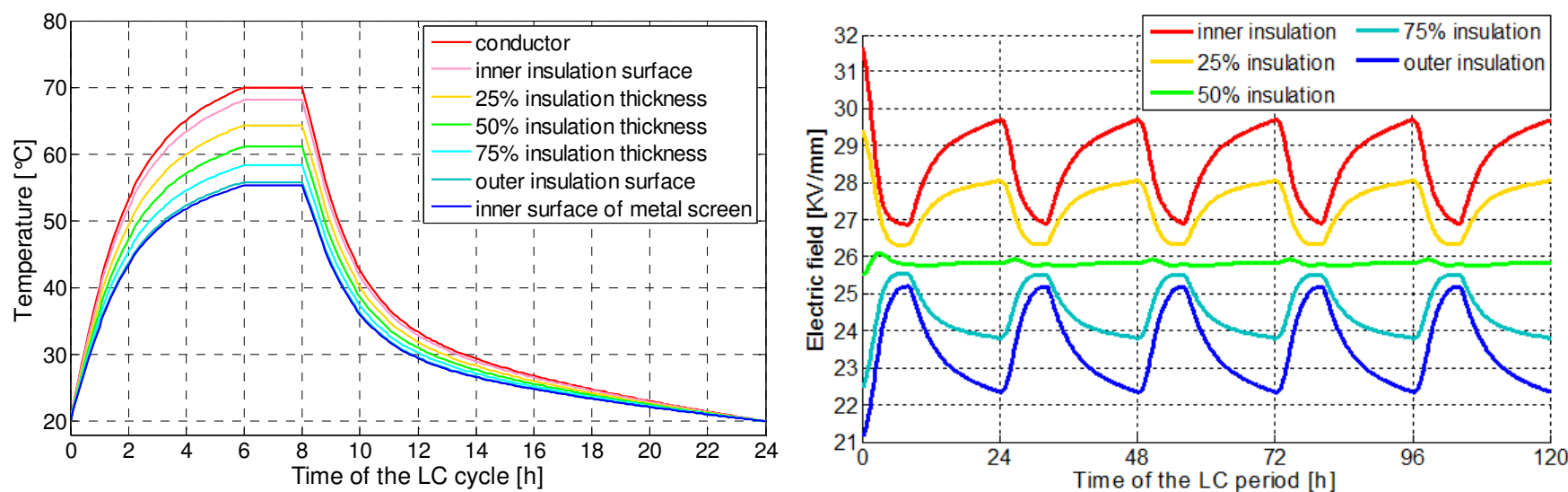

Figure 3. Transient temperatures during the load Figure 4. Transient exact electric fields during the cycles of the LC period [9] at 7 points in the sample first 5 load cycles of the LC period at 5 points in the cable. insulation of the sample cable for $a_{L}, b_{L}$.
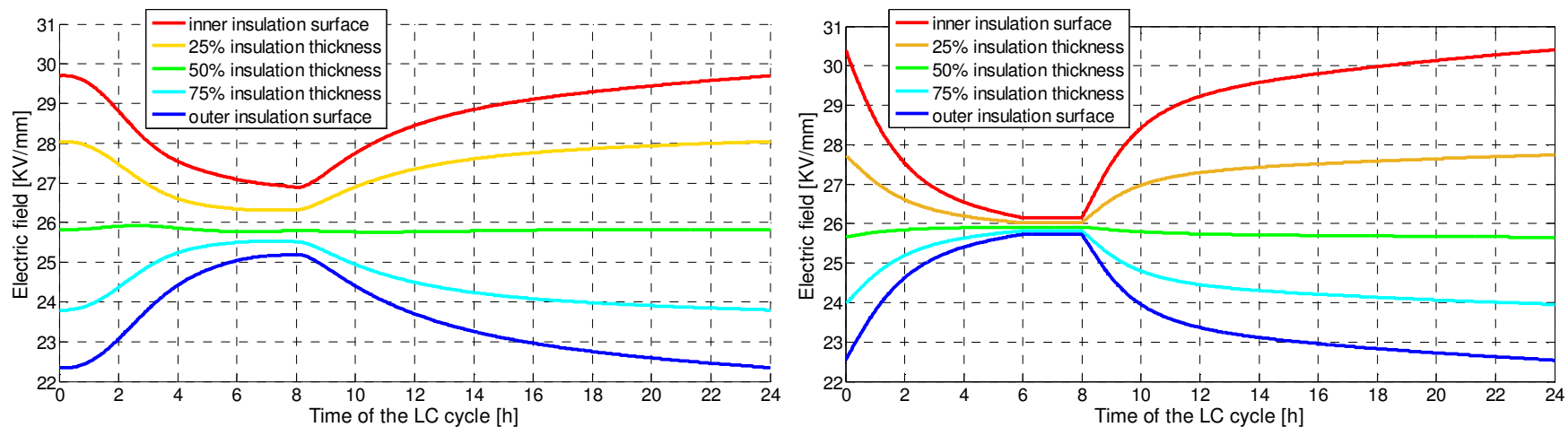

a)

b)

Figure 5. Transient electric fields for the sample cable during the daily load cycles of LC period at 5 locations in the insulation for $a_{L}, b_{L}$ a) exact field, $3^{\text {rd }}$ cycle (and following cycles); b) approximate field (all cycles).

cause the approximate field according to Eoll's formula is the steady-state DC electric field at a given temperature.

Comparison of Figures 5 (a) and (b) also shows that

(1) in the hottest part of the LC (between 6 and $8 \mathrm{~h}$ ) the approximate profiles are closer to each other than the exact profiles. Since field inversion does not occur, it follows that the exact field is higher than the approximate field at the inner surface of the insulation, and lower at the outer surface

(2) the opposite is true in the coldest part of the LC $(0 \mathrm{~h}$ and between $22 \mathrm{~h}$ and $24 \mathrm{~h})$

(3) at $t=0 \mathrm{~h}$ (cold cable), and at the inner insulation surface, the exact and approximate fields (red curves in Figures 5(a) and (b) respectively) are the highest over the complete cycle and within the insulation. They drop as the cable is heated, until the conductor temperature reaches $T_{D}=70^{\circ} \mathrm{C}$ (Fig. 3). Throughout the hottest part of the LC cycle $(6-8 \mathrm{~h})$ the approximate field is stable, and the exact field drops only slightly. Both fields then increase to the no-load maximum as the cable is cooled down.

(4) at the outer insulation surface the behavior of the exact and approximate fields is the opposite of that at the inner insulation surface.

(5) at intermediate points within the insulation, the approximate and exact fields lie between the fields at the inner and outer insulation surfaces, as expected. Both transient fields at 50\% insulation thickness are very nearly constant throughout the daily LC, in agreement with Figure 2.

The points (1) to (5) above are noteworthy because they are essentially consequences of the absence of field inversion in the sample cable, at the chosen values of $a_{L}, b_{L}$. The detailed qualitative and quantitative 


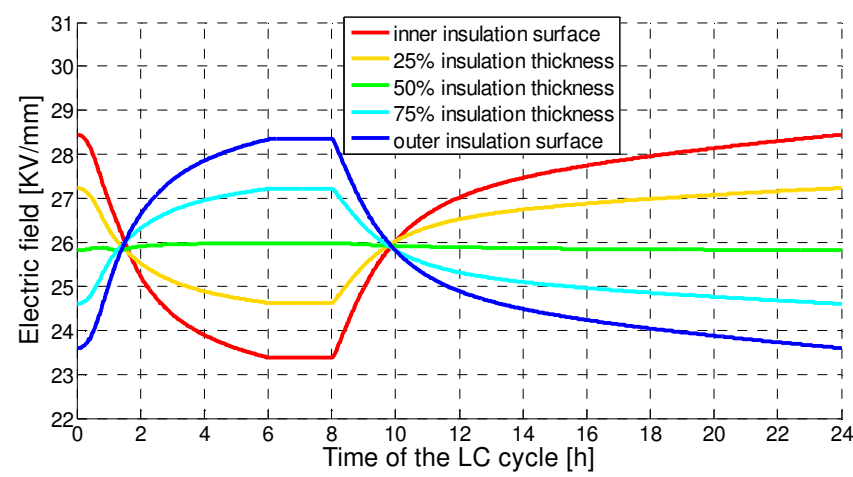

a)

Figure 6. Transient electric fields for the sample cable during the daily LC at 5 locations in the insulation for $\left.a_{H}, b_{H} . a\right)$ exact field in the $3^{\text {rd }}$ cycle; $b$ ) approximate field (all cycles).

effects of the absence of field inversion on the electric field profiles cannot be predicted theoretically a priori. They can be assessed only after the calculations have been made using the original and improved versions of the procedure.

The exact and approximate field profiles are also very sensitive to the $a, b$ values, as seen by comparing Figures 5(a) and (b) with Figures 6(a) and (b), the former obtained using $a_{L}, b_{L}$ and the latter using $a_{H}, b_{H}$ (Table 1). Similar sensitivity was observed using $a_{M}, b_{M}$ (omitted here for brevity). For both the exact and approximate field profiles, field inversion occurs for $a_{H}, b_{H}$ and $a_{M}, b_{M}$, i.e. the field in the hottest part of the LC $(6-8 \mathrm{~h})$ is higher at the outer than at the inner insulation surface. The higher $a$ and $b$, the stronger the inversion, i.e., the field at the outer insulation surface increases and the field at the inner insulation surface decreases.

Figures 6(a) and (b) also show that

(1) at the inner and outer insulation surfaces, both the exact and the approximate field profiles reach steady state within the hot part of the LC. This happens because $a_{H}, b_{H}$ imply higher conductivity and thus lower dielectric time constants $(\tau=0.01-0.02 \mathrm{~h})$ and shorter time to steady state $(\tau=0.1-0.2 \mathrm{~h})$ than $a_{L}, b_{L}$

(2) in the hottest part of the LC $(6-8 \mathrm{~h})$ the exact field profiles within the insulation are closer to each other than are the approximate field profiles. Since field inversion occurs, it follows that the exact field is higher than the approximate field at the inner surface of the insulation, and lower at the outer surface.

(3) in the coldest part of the LC $(0 \mathrm{~h}, 22-24 \mathrm{~h})$ the approximate field profiles are closer to each other than exact field profiles. Since field inversion does occur, it follows that the exact field is lower than the approximate field at the inner surface of the insulation and at intermediate points up to the mid-point of the insulation, and lower from the mid-point to the outer surface.

The points (1) to (3) above are noteworthy because they are essentially consequences of field inversion in the sample cable, at the chosen values of $a_{H}, b_{H}$. The detailed qualitative and quantitative effects of the field inversion on the electric field profiles cannot be predicted theoretically a priori. They can be assessed only after the calculations have been made using the original and improved versions of the procedure.

The approximate field profiles are constant throughout the HL and ZL periods. On the other hand the exact field profiles are changing at the beginning of these periods, passing from the purely-capacitive (AC) distribution at the start to the purely-resistive (DC) distribution at the end. A steady state is reached sooner in the HL period than in the ZL period, because temperature and conductivity are higher in the former. A steady state is also reached sooner at higher $a, b$, since higher $a, b$ imply higher conductivity. The profiles in the HL and ZL periods show the same features as those in the hot and cold parts respectively of the LC.

\section{Block 4: Calculation of Loss-of-life Fractions and DC Cable Life Estimation}

The loss-of-life fractions $L F_{D C}(r)$ of the cable insulation at every radius $r$ were computed using (1) for the LC, HL, ZL periods of the PQ tests. Details of the calculation of $L F_{D C}(r)$ for the exact and approximate field, in the HL and ZL periods, are omitted here, because such calculations are straight forward. 
On the contrary, the computation of the loss-of-life fractions $L F_{D C}(r)$ is much more complex for the daily load cycles of the LC period. Such loss-of-life fractions for the sample cable at the same 5 locations within the insulation as in Figure 5, calculated using the exact and approximate field profiles with $a_{L}, b_{L}$ are presented in Figure 7(a) and (b) respectively. These Figures show that

(1) In all three steps of LC cycles (step $\Delta t_{1}$ from $0 \mathrm{~h}$ to $6 \mathrm{~h}$, step $\Delta t_{2}$ from 6 to $8 \mathrm{~h}$, step $\Delta t_{3}$ from 8 to $24 \mathrm{~h}$ ) the highest values of $L F_{D C}(r)$ occur at the inner insulation surface, and the lowest values at the outer insulation surface. This is consistent with the temperature profiles in Figure 3 and the field profiles in Figures 4 and 5. Figures 3-5 show that, during each of the three steps of the LC cycles, the inner insulation surface is the most stressed point, with the highest fields and temperatures and the highest loss-of-life fractions, the outer insulation surface is the least stressed point with the lowest fields and temperatures and the lowest loss-of-life fractions, and the intermediate points have intermediate loss-of-life fractions.

(2) At all points within the cable insulation the highest values of $L F_{D C}(r)$ occurred in the $1^{\text {st }}$ step $(0-6 \mathrm{~h}$, blue bars in Figure 7(a) and Figure 7(b)), followed by the 2nd step (6-8 h, green bars) and 3rd step (8-24 h, brown bars). This ordering is to be expected. Figure 3 shows that the 3rd step can be regarded as a "cold" step during which the temperature drops rapidly $\left(>40^{\circ} \mathrm{C}\right.$ in the whole insulation for less than $\left.2 \mathrm{~h}\right)$ and has a lesser effect than in the other steps, independent of the $a, b$ values. Conversely the 1st step can be regarded as a "hot" step during which the temperature rises rapidly $\left(>40^{\circ} \mathrm{C}\right.$ in the whole insulation for more than 4 hours). The 2 nd step has values of $L F_{D C}(r)$ lower but comparable to the 1st step, as temperature in the 2nd step is always the highest over the entire insulation. This ordering implies that the loss-of-life fraction over the whole PQ test will be affected greatly during the HL period, where the insulation is always at the highest temperature, less affected during the LC period, which is the longest but includes hot and colt parts, and negligibly affected during the ZL period, where the insulation is always at the lowest temperature (room temperature).

(3) at the inner insulation surface the $L F_{D C}(r)$ values in the $1^{\text {st }}$ and $2^{\text {nd }}$ steps are approximately $30 \%$ higher for the exact field than for the approximate field. This is consistent with the field profiles in Figures 5(a) and 5(b). At the inner insulation surface, the most electrically stressed point, the exact field is higher than the approximate field in the hot part of the $1^{\text {st }}$ step and in the $2^{\text {nd }}$ step of LC cycles.

(4) at the outer insulation surface the loss-of-life fractions are significantly lower for the exact than for the approximate field. Again, this is consistent with the field profiles in Figures 5(a) and 5(b). At the outer insulation surface, the least electrically stressed point, the exact field is lower than the approximate field in the hot part of the $1^{\text {st }}$ and $2^{\text {nd }}$ steps, making the loss-of-life fractions significantly lower for the exact field than for the approximate field in these steps.

(5) at intermediate points the loss-of-life fractions are about the same for the exact and approximate fields, to be expected because the relevant field profiles in Figures 5(a) and 5(b) are very similar for the exact and approximate fields.

(6) The loss of life fraction over the whole PQ test is expected to be significantly higher for the exact field than for the approximate field at the inner insulation surface, significantly higher for the approximate field than for the exact field at the outer insulation surface, and about the same for both fields at the mid-point of the insulation, as shown in Figures 7(c) and (d).

Figures 7(c) and 7(d) show respectively the loss-of-life fractions calculated using the exact and approximate field for the sample cable, over the complete ZL, HL and LC periods and the whole PQ test, at the same 5 locations within the insulation as in Figure 6, and for $a_{L}, b_{L}$. They show that

(a) the highest loss-of-life fractions over the whole PQ test occur at the inner insulation surface, the most stressed point 

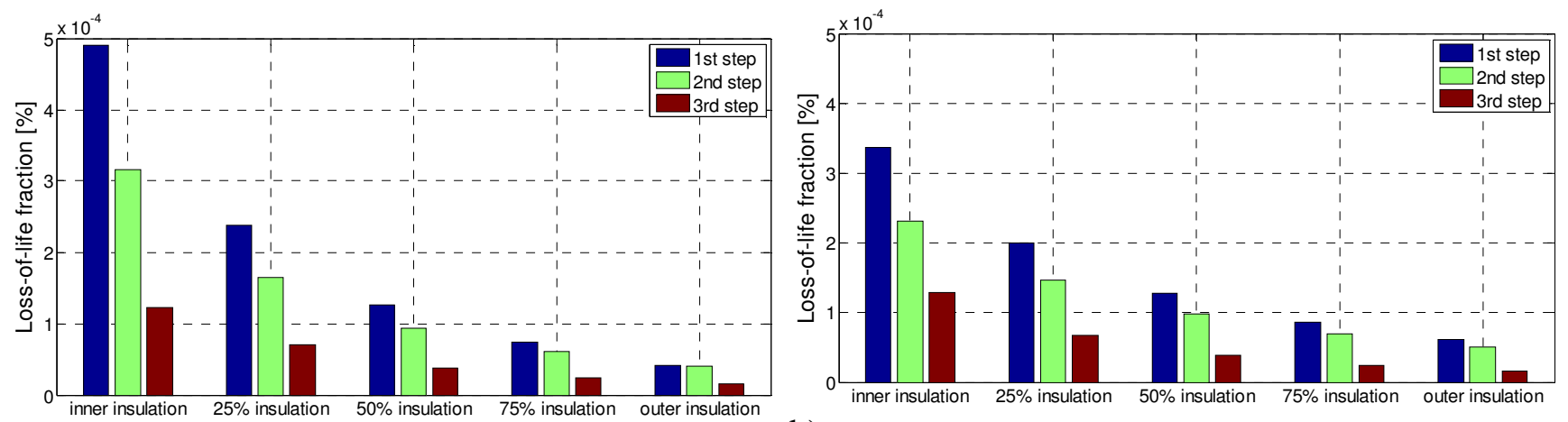

a)

b)
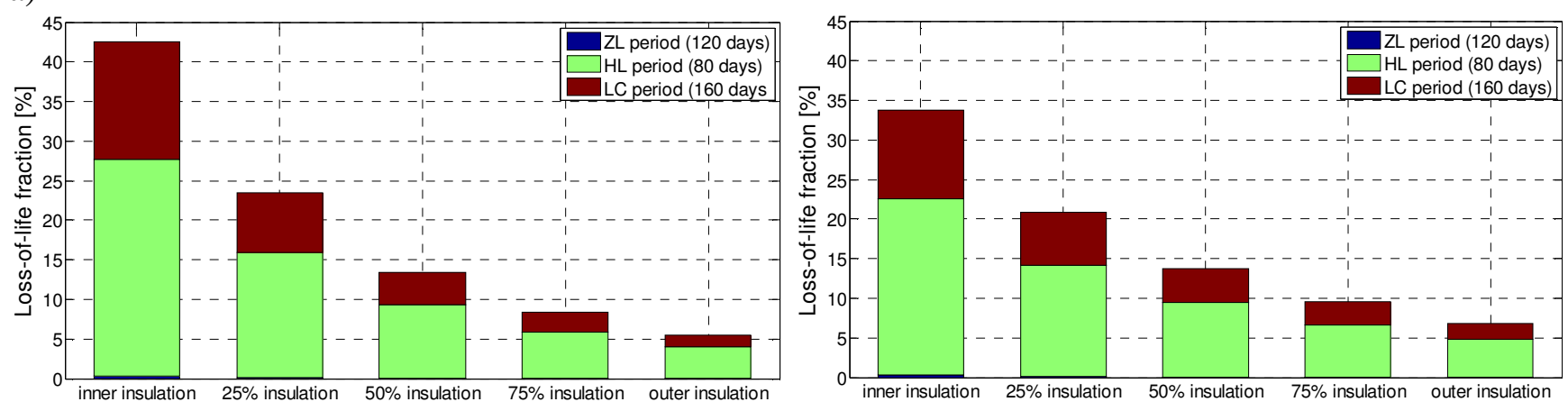

c)

d)

Figure 7. Loss-of-life fractions for the sample cable at 5 locations in the insulation using $a_{L}, b_{L}$.

(a) in the three steps of a generic 24 hour cycle of the LC for exact field profiles

(b) in the three steps of a generic 24 hour cycle of the LC for approximate field profiles

(c) in the $L C, H L, Z L$ periods, and in the 360 day $P Q$ tests (= sum of $L C, H L$ and $Z L)$ for exact field profiles

(d) in the $L C, H L, Z L$ periods, and in the 360 day $P Q$ tests (= sum of $L C, H L$ and $Z L$ ) for approximate field profiles.

(b) as expected from (2) immediately above, the loss-of-life is highest in the HL period (green part of bars), lower in the LC period (brown part of bars), and negligible in the ZL period (blue part of bars)

(c) as expected from (6) immediately above, over the whole PQ test (whole bars) the loss-of-life is significantly higher at the inner insulation surface for the exact field, and at the outer insulation surface for the approximate field. Since the inner insulation is by far the most stressed point, the PQ life would be expected to be shorter for the exact field.

Figures 8(a) and (b) show data corresponding to those in Figures 7(a) and (b), but for $a_{H}, b_{H}$. These Figures show that

(1) in the 1st and 2nd steps of the LC periods, for both the exact and approximate fields, the highest lossof-life fractions occur at the outer insulation surface, and the lowest at the inner insulation surface. This is consistent with the temperature profiles in Figure 3 and the field profiles in Figures 6(a) and (b). The latter show that, in the 1st and 2nd steps the outer insulation surface is on the whole the most stressed point of the insulation because of the very strong field inversion during the hotter part of these steps (in which the conductor temperature is mostly close to or equal to $T_{D}$ ). Conversely the inner insulation surface is on the whole the least stressed point of the insulation. Figures 3, 6(a) and (b) also show that, in the $3^{\text {rd }}$ step of the LC, the highest loss-of- life fractions occur at the inner insulation surface. This is consistent with the fact that the $3^{\text {rd }}$ step is dominated by its cold part, during which the inner insulation surface is the most stressed point. 
(2) at all points within the cable insulation, for both the exact and approximate fields, the highest loss-oflife fractions occur in the $1^{\text {st }}$ step, followed by those in the 2 nd and 3rd steps. Again, this is due to temperature, as for Figures 7(a) and (b). It follows that the loss-of-life fraction over the whole PQ test will be affected greatly by the HL period, corresponding to the highest temperatures in the insulation, less affected by the LC period with its hot and cold parts, and negligibly affected by the ZL period, with the lowest insulation temperatures.

(3) at the outer insulation surface the loss-of-life fractions are lower for the exact than for the approximate field, in the range $10-15 \%$. Conversely, the loss-of-life fractions at the inner insulation surface are higher for the exact than for the approximate field, in the range 9-22\%. At mid-insulation the loss-of-life fractions with the exact and with the approximate field are about the same. This is consistent with the field profiles in Figures 6(a) and (b).

(4) It follows from (3) immediately above that the loss-of-life in the whole PQ test will be significantly higher at the inner insulation surface for the exact field, and at the outer insulation surface for the approximate field.

Figures 8(c) and 8(d) show data corresponding to those in Figures 7(c) and (d), but for $a_{H}, b_{H}$. They show that:

(1) the highest loss-of-life fractions occur at the outer insulation, the most stressed point over the whole PQ test

(2) as expected from item (2) of the observations on Figures 8(a),(b), the loss-of-life fractions are highest in the HL period, lower in the LC period, and negligible in the ZL period

(3) as expected from item (4) of the observations on Figures 8(a),(b), over the complete PQ test the loss-oflife fraction is significantly higher at the inner insulation surface for the exact field, and at the outer insulation surface for the approximate field. Since the outer insulation is by far the most stressed point when using $a_{H}, b_{H}$, the PQ life is expected to be higher for the exact field.

Block No. 4 is now completed by computing:

(a) the number of cycles to failure at every radius $r, K_{D C}(r)=1 / L F_{D C}(r)$, using (1)

(b) the insulation life under PQ load cycles at every radius $r, L_{D C}(r) \propto K_{D C}(r)$, using (2)

(c) the cable life under load cycles, $L_{D C}=\min \left\{L_{D C}(r)\right\}$, using (3).

As shown in Figures 7(c) and (d), and in Figures 8(c) and (d), the loss-of-life fractions over the whole PQ test are all $\ll 100 \%$, even at the most highly stressed points. Thus the PQ life will be longer than the PQ test duration (=360 days) at all points within the insulation, and for all three $a, b$ sets. This is confirmed by the values of $L_{D C}(r)$ listed in Table 2 for each of the three $a, b$ sets. $L_{D C}$ is the PQ life, i.e., the minimum value of $L_{D C}(r)$ at the most stressed point, denoted by a gray-shaded cell.

\section{Discussion and Conclusions}

Table 2 shows that the improved procedure based on the exact field, and the earlier procedure based on approximate field [13], yield PQ life estimates much longer than the PQ test duration. Thus both procedures suggest that the PQ test with maximum conductor temperature set exactly to $T_{D}$ is not sufficiently stressful for cable insulation testing. This finding might be expected, since thermal aging and electrothermal synergism are neglected in CIGRE brochure 496 [9].

All the other results provided by the two procedures differ significantly, e.g., transient field profiles (Figures 4-6), loss-of-life fractions over the LC, HL, ZL periods and the complete PQ tests (Figures 7-8), and most importantly the PQ life (Table 2). Table 2 also shows that for $a_{M}, b_{M}$ the exact field yields the minimum value of $L_{D C}(r)$ at the inner insulation surface, while the approximate field yields the minimum value of $L_{D C}(r)$ at the outer insulation surface. Although details of field profiles and life fractions for $a_{M}, b_{M}$ were omitted for the sake of brevity, it can be said that the differences between the locations of the minimum $L_{D C}(r)$ stem from the moderate field inversion occurring for $a_{M}, b_{M}$ and from the small, but nonnegligible differences between the exact and approximate field profiles occurring under $a_{M}, b_{M}$. 

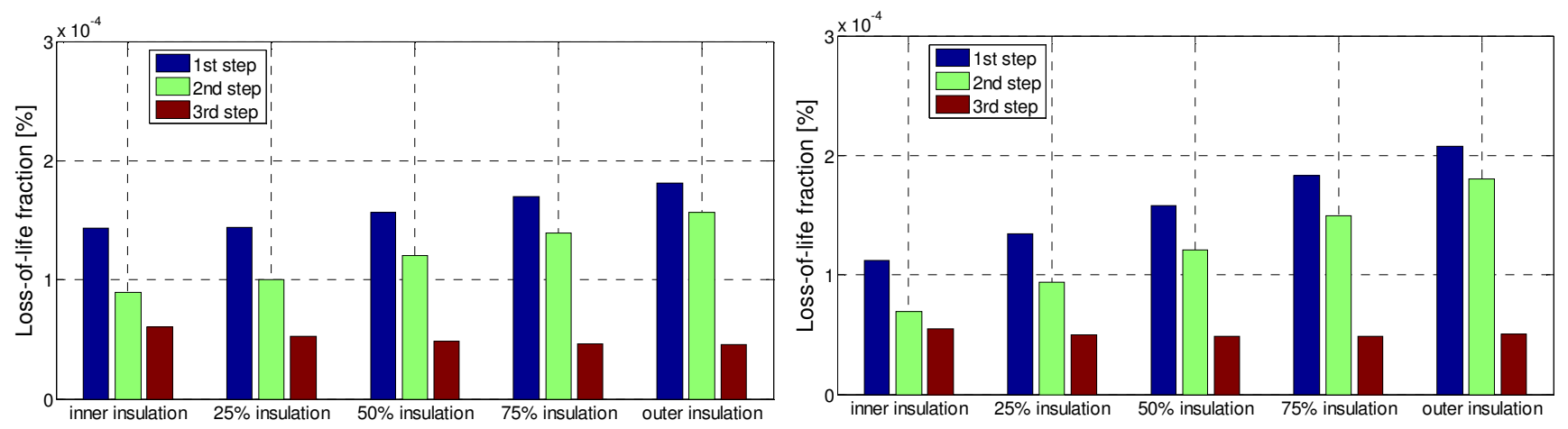

a)

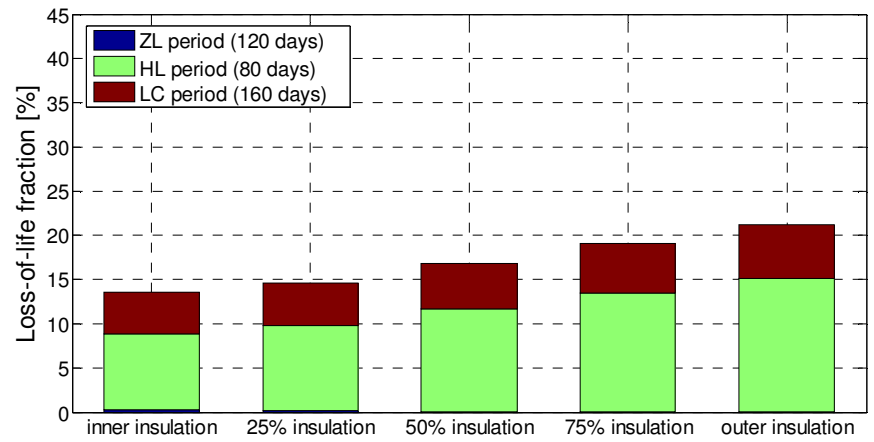

b)

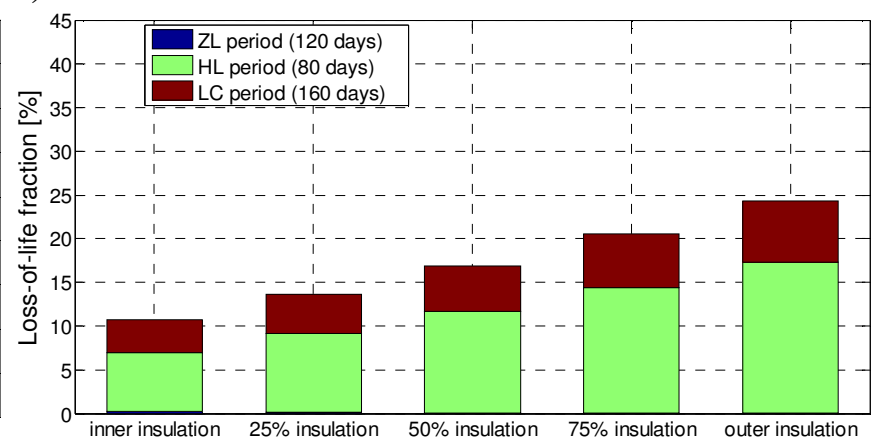

c)

d)

Figure 8. Loss-of-life fractions for the sample cable at 5 locations in the insulation using $a_{H}, b_{H}$.

(a) in the three steps of a generic 24 hour cycle of the LC for exact field profiles

(b) in the three steps of a generic 24 hour cycle of the $L C$ for approximate field profiles

(c) in the $L C, H L, Z L$ periods, and in the 360 day $P Q$ tests (= sum of $L C, H L$ and $Z L)$ for exact field profiles

(d) in the $L C, H L, Z L$ periods, and in the 360 day $P Q$ tests (= sum of $L C, H L$ and $Z L$ ) for approximate field profiles.

Table 2. Values of insulation life $L_{D C}(r)$ computed with the exact field and approximate field profiles for $a_{L}, b_{L}$, $a_{M}, b_{M}$, and $a_{H}, b_{H}$, at 5 locations within the insulation of the sample cable. $L_{D C}$ is the $P Q$ life, i.e., the minimum value of $L_{D C}(r)$ at the most stressed point, denoted by a gray-shaded cell.

\begin{tabular}{|c|c|c|c|c|c|c|}
\hline \multirow{2}{*}{$\begin{array}{c}a, b \text { values } \\
\text { Location }\end{array}$} & \multicolumn{2}{|c|}{$a_{L}, b_{L}$} & \multicolumn{2}{c|}{$L_{D C}(r)[\mathrm{y}]$} \\
\cline { 2 - 7 } & exact field & approx. field & exact field & approx. field & exact field & $a_{H}, b_{H}$ \\
\hline inner insulation & 2.32 & 2.93 & 4.19 & 5.32 & 7.29 & 9.22 \\
\hline $25 \%$ insulation & 4.20 & 4.73 & 4.47 & 4.82 & 6.78 & 7.24 \\
\hline $50 \%$ insulation & 7.32 & 7.17 & 4.44 & 4.39 & 5.86 & 5.83 \\
\hline $75 \%$ insulation & 11.6 & 10.3 & 4.39 & 4.05 & 5.17 & 4.80 \\
\hline outer insulation & 17.8 & 14.4 & 4.35 & 3.77 & 4.64 & 4.05 \\
\hline
\end{tabular}

The improved procedure presented here is more flexible than might appear at first sight, since the expressions chosen for the electro-thermal life model and the electrical conductivity can be replaced with others which fit better the particular type of DC insulation being studied.

It should be noted that the improved procedure contains an important simplifying hypothesis, i.e., inhomogeneities within the dielectric can be neglected. Such inhomogeneities may cause spatial variations of the temperature and field coefficients of the volume electrical conductivity, and highly-localized space charge distributions sometimes reported [30],[31]. 


\section{References}

[1] G. Mazzanti, M. Marzinotto, Extruded Cables for High Voltage Direct Current Transmission: Advances in Research and Development, Power Engineering Series. Wiley-IEEE Press, 2013.

[2] J. Fothergill, "The coming of age of HVDC extruded power cables", in 2014 IEEE Electr. Insul. Conf., 2014, pp. $124-137$.

[3] M. Albertini, A. Bareggi, L. Caimi, L. De Rai, A. Dumont, Stefano Franchi Bononi, Giovanni Pozzati, and Paolo Boffi, "Development and high temperature qualification of innovative $320 \mathrm{kV}$ DC cable with superiorly stable insulation system", in $9^{\text {th }}$ Int. Conf. Insul. Power Cables (Jicable'15), 2015, Paper A7.3.

[4] http://b4.cigre.org/Publications/Other-Documents/Compendium-of-all-HVDC-projects/

[5] P. Labra Francos, S. Sanz Verdugo, and S. Guyomarch, "New French-Spanish VSC link", CIGRÉ Session 2012, paper B4110, pp. 1-15, 2012.

[6] T. Katayama, Y. Murata, T. Yamazaki, T. Igi, S. Kashiyama, and M. Watanabe, "Completion of $400 \mathrm{kV}$ pre-qualification test for DC-XLPE cable system, in Jicable HVDC 2013, 2013, Paper P16.

[7] A. Gustafsson, M. Jeroense, H. Ghorbani, T. Quist, M. Saltzer, A. Farkas, F. Axelsson, and V. Mondiet, "Qualification of an extruded HVDC cable system at $525 \mathrm{kV}$ ", in $9^{\text {th }}$ Int. Conf. Insul. Power Cables (Jicable'15), 2015, Paper A7.1.

[8] G. Mazzanti and M. Marzinotto, "Advanced electro-thermal life and reliability model for high voltage cable systems including accessories”, IEEE Electr. Insul. Mag., vol. 33, no. 3, pp. 17-25, May/Jun. 2017.

[9] CIGRÉ WG B1.32, "Recommendations for testing DC extruded cable systems for power transmission at a rated voltage up to 500kV”, Brochure CIGRÉ 496, Apr. 2012.

[10] M. Marzinotto, G. Mazzanti, and C. Mazzetti, "A new approach to the statistical enlargement law for comparing the breakdown performance of power cables - Part 1: theory", IEEE Trans. Dielectr. Electr. Insul., pp. 1232-1241, vol.14, n.5, 2007.

[11] T.L. Hanley, R.P. Burford, R.J. Fleming, and K.W. Barber, "A general review of polymeric insulation for use in HVDC cables", IEEE Electr. Insul. Mag., vol. 19, no. 1, pp. 14-24, Jan./Feb. 2003.

[12] R.N. Hampton, "Some of the considerations for materials operating under high-voltage, direct-current stresses", IEEE Electr. Insul. Mag., vol. 24, no. 1, pp. 5-13, Jan./Feb. 2008.

[13] G. Mazzanti, "Life estimation of HVDC cables under the time-varying electro-thermal stress associated with load cycles", IEEE Trans. Power Del., vol. 30, no. 2, pp. 931-939, 2015.

[14] C.K. Eoll, "Theory of stress distribution in insulation of high voltage d.c. cables. Part I", IEEE Trans. Electr. Insul., vol. EI10, no. 1, pp. 27-35, 1975.

[15] M.J.P. Jeroense and P.H.F. Morshuis, "Electric fields in HVDC paper-insulated cables", IEEE Trans. Dielectr. Electr. Insul., vol. 5, no. 2, pp. 225-236, 1998.

[16] G. Mazzanti, "Analysis of the combined effects of load cycling, thermal transients and electro-thermal stress on life expectancy of high voltage ac cables", IEEE Trans. Power Del., vol. 22, no. 4, pp. 2000-2009, 2007.

[17] G. Mazzanti, "The combination of electrothermal stress, load cycling and thermal transients and its effects on the life of high voltage ac cables", IEEE Trans. Dielectr. Electr. Insul., vol. 16, no. 3, pp. 1168-1179, 2009.

[18] Calculation of the Cyclic and Emergency Current Rating of Cables, Part2: Cyclic Rating of Cables Greater Than 18/30 (36) $\mathrm{kV}$ and Emergency Ratings for Cables of All Voltages, Standard IEC 60853-2, Ed. 1.0, Jan. 1989.

[19] M. A. Miner, "Cumulative damage in fatigue", J. Appl. Mechanics, pp. A159-A163, Sep. 1945.

[20] G. Mazzanti, G. C. Montanari, and F. Civenni, "Model of inception and growth of damage from microvoids in polyethylene-based materials for HVDC cables - Part 2: Parametric investigation and data fitting", IEEE Trans. Dielectr. Electr. Insul., pp. 1255-1263, vol. 14, no. 5, 2007.

[21] G. Mazzanti, G.C. Montanari, and L. Simoni, "Insulation characterization in multistress conditions by accelerated life tests: an application to XLPE and EPR for high-voltage cables”, IEEE Electr. Insul. Mag., vol. 13, no. 6, pp. 24-33, 1997.

[22] Electrical Insulating Materials - Properties of Thermal Endurance - Part 1: Ageing Procedures and Evaluation of Test Results, Standard IEC 60216-1, Ed. 5.0, Jul. 2001.

[23]E. Chiodo and G. Mazzanti, "Theoretical and practical aids for the proper selection of reliability models for power system components", Int. J. Reliabil. Safety, vol. 2, no. 1/2, pp. 99-128, 2008.

[24] L.A. Dissado and J.C. Fothergill, Electrical Degradation and Breakdown in Polymers. Peregrinus, London, 1992.

[25] G. Chen, T.Y. G. Tay, A.E. Davies, Y. Tanaka, and T. Takada, "Electrodes and charge injection in low-density polyethylene using the pulsed electroacoustic technique", IEEE Trans. Dielectr. Electr. Insul., vol. 8, no. 6, pp. 867-873, 2001.

[26] S. Le Roy, G. Teyssedre, C. Laurent, G C Montanari, and F Palmieri, "Description of charge transport in polyethylene using a fluid model with a constant mobility: fitting model and experiments”, J. Phys.D: Appl.Phys., Vol.39, pp.1427-1436, 2006.

[27] C.M. Snowden, R.E. Miles, Compound Semiconductor Device Modelling, Springer Science \& Business Media, Dec. 2012.

[28] H. J. Wintle, "Charge motion in technical insulators: facts, fancies and simulations", IEEE Trans. Dielectr. Electr. Insul., vol. 10, no. 5, pp. 826-841, 2003.

[29] Electric cables - calculation of the current rating - Part 1-1: current rating equations (100\% load factor) and calculation of losses - General", Standard IEC 60287-1-1, Ed. 2.0, Dec. 2006.

[30] T. Takada, "Acoustic and optical methods for measuring electric charge distributions in dielectrics", IEEE Trans. Dielectr. Electr. Insul., vol. 6, no. 5, pp. 519-547, 1999. 
[31] G. Mazzanti, G. Chen, J. Fothergill, N. Hozumi, J. Li, M. Marzinotto, F. Mauseth, P. Morshuis, A. Tzimas, C. Reed, and K. Wu, "A protocol for space charge measurements in full-size HVDC extruded cables", IEEE Trans. Dielectr. Electr. Insul., vol. 22, no. 1, pp. 21-34, 2015.

[32] G. Mazzanti, G.C. Montanari, and L. Simoni, "Study of the Synergistic Effect of Electrical and Thermal Stresses on Insulation Life", in Conf. Record of 1996 Conference on Electrical Insulation and Dielectric Phenomena (CEIDP), pp. 684-687, San Francisco, California (USA), 20-23 Oct. 1996

[33] S. V. Patankar, Numerical Heat Transfer and Fluid Flow, Taylor \& Francis, 1980. ISBN 978-0-89116-522-4.

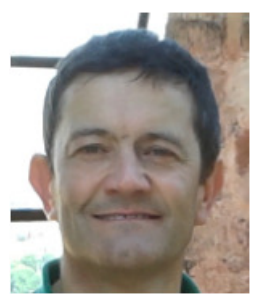

Giovanni Mazzanti (M '04 SM '15) is Associate Professor of HV Engineering and Power Quality at the University of Bologna. His research interests are life modeling, reliability and diagnostics of HV insulation, power quality, renewables and human exposure to electromagnetic fields. He is consultant to TERNA (the Italian TSO) in the HVDC and HVAC cable systems area, E-Distribuzione (the main Italian DSO) and CESI S.p.A. He is author or coauthor of more than 250 published papers, and coauthor of the book Extruded Cables for High Voltage Direct Current Transmission: Advances in Research and Development, John Wiley-IEEE Press, 2013. He is Senior Member of IEEE, and chairman of the IEEE DEIS Technical Committee/Working Group "HVDC cable systems (cables, joints and terminations)". 